\title{
Analysis of the flow imbalance on the profile shape during the extrusion of thin magnesium sheets
}

Cite as: AIP Conference Proceedings 1567, 1098 (2013); https://doi.org/10.1063/1.4850162

Published Online: 31 December 2013

Sven Gall, Sören Müller, and Walter Reimers

\section{ARTICLES YOU MAY BE INTERESTED IN}

Electrohydraulic forming of dual phase steels; numerical and experimental work AIP Conference Proceedings 1567, 1115 (2013); https://doi.org/10.1063/1.4850166

Prediction of particle orientation in simple upsetting process of $\mathrm{NdFeB}$ magnets AIP Conference Proceedings 1567, 1119 (2013); https:/ / doi.org/10.1063/1.4850167

Study on bulging behavior of rotating cylinder compression forming based on upper bound method and experiment

AIP Conference Proceedings 1567, 1103 (2013); https://doi.org/10.1063/1.4850163

\section{Lock-in Amplifiers up to $600 \mathrm{MHz}$

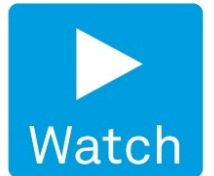

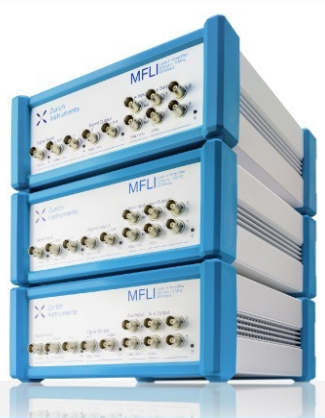




\title{
Analysis Of The Flow Imbalance On The Profile Shape During The Extrusion Of Thin Magnesium Sheets
}

\author{
Sven Gall ${ }^{\mathrm{a}, \mathrm{b}}$, Sören Müller ${ }^{\mathrm{a}}$, Walter Reimers ${ }^{\mathrm{b}}$ \\ ${ }^{a}$ Forschungszentrum Strangpressen, Technische Universität Berlin, Gustav-Meyer-Allee 25, Berlin, 13355, \\ Germany, ${ }^{b}$ Metallische Werkstoffe, Technische Universität Berlin, Ernst-Reuter-Platz 1, Berlin, 10587, Germany
}

\begin{abstract}
The extrusion process facilitates the production of magnesium sheets featuring a very thin thickness as well as excellent surface properties by using a single process step only. However, the extrusion of the magnesium sheets applying not optimized process parameters, e.g. low billet temperature or/ and poorly deformable magnesium alloy, produce pronounced buckling and waving of the extruded sheets as well as a variation of accuracy in profile shape along the cross section. The present investigation focuses on the FEM-simulation of the extrusion of magnesium sheets in order to clarify the origin of the mentioned effects. The simulations identify the flow imbalance during extrusion as the main critical factor. Due to the flow imbalance after passing the die a large compression stress zone is formed causing the buckling and waving of the thin sheets. Furthermore, the simulations of the magnesium sheet extrusion reveal that the interaction of the material flow gradients along the width and along the thickness direction near the die orifice lead to the variation of the accuracy in profile shape.
\end{abstract}

Keywords: extrusion, FE-simulation, flow imbalance, magnesium.

PACS: $81.20 . \mathrm{Hy}$

\section{INTRODUCTION}

Magnesium alloys are continuously of interest for lightweight applications due to their high specific strength and stiffness. However, they possess poor formability due to their crystallographic structure delivering limited number of slip systems at ambient temperature. Thus, the magnesium production is mostly conducted by casting processes. In order to widen the field of magnesium applications an increasing employment of wrought alloys for rolling, extrusion and forging is necessary $[1,2]$. Flat products are usually manufactured by rolling processes using multiple rolling as well as reheating steps between the rolling passes. In contrast, the extrusion process facilitates the production of flat rectangular profiles using a single forming step only [3]. Applying optimized process parameters the extruded sheets feature excellent surface properties without any defects. However, when using not optimized extrusion process parameters, e.g. low billet temperature, high extrusion speed, large extrusion ratio as well as poorly deformable magnesium alloys variances in profile shape and pronounced buckling and waving of the sheets arise (FIGURE 1).

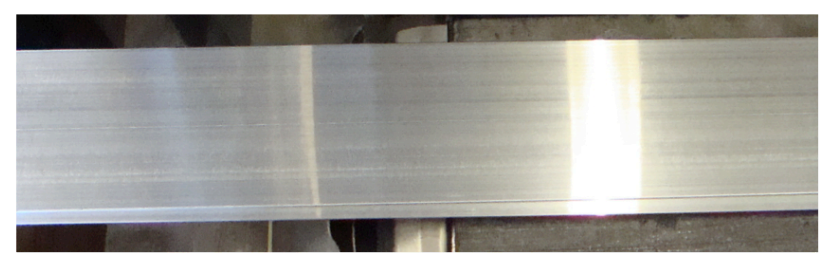

Flat profile featuring excellent surface quality

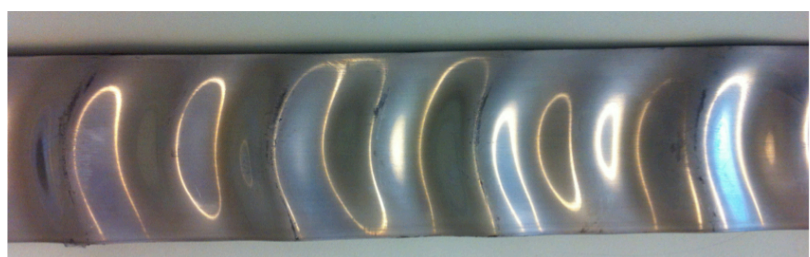

Flat profile revealing pronounced buckling

FIGURE 1. Directly extruded flat profiles of the magnesium alloy ME21

Sheets produced by extrusion are usually the entry material for downstream forming processes such as deep drawing or stretch bending. Beside the surface quality and the mechanical properties the accuracy in profile shape is important for successful forming. Variances in profile geometry restrict the forming process and may lead to defects on the final product. Therefore, the extrusion of sheets revealing geometries within narrow tolerances is required. In order to meet the requested tolerances, it is necessary to understand and control the underlying mechanisms causing 
profile shape variations. In this context, FE-simulations are a very useful tool to identify and localize the critical variables leading to the mentioned phenomena [4].

The present work focuses on the identification and understanding of the buckling and the variances in profile shape of flat thin magnesium strips during extrusion utilizing FE-simulations. The analysis of the stress, velocity, strain and temperature distributions will identify the underlying mechanisms causing shape variations in the magnesium extrusions.

\section{MODEL DESCRIPTION}

FE-simulation is conducted using the commercial FEM-software package HyperXtrude ${ }^{\circledR}$ by Altair Engineering. It is based on the fluid-dynamic formulation that adopts the ALE-(Arbitrary Lagrangian Eulerian) approach and was chosen due to shorten the computation time since time-consuming re-meshing steps are significantly reduced in comparison to FE-software based on the Lagrangian approach. However, in consequence of the ALE-formulation in the simulation it is not possible to consider all extrusion components such as the stem, dummy block and container, which have to be idealized by appropriate boundary conditions.

FIGURE 2 illustrates the global model of the direct magnesium sheet extrusion. The FE-model contains the billet, the die and the profile (please note the FE-model and all simulation parameters correspond to the experimental study published in [3]). Furthermore, the profile is divided in a section, in which the material having die contact (bearing material) as well as in a profile section without die contact (exit profile). Due to the fine meshing especially of the deformation zone, the very thin profile strips and the bearing a FE-model considering $1 / 4^{-}$ symmetry was generated in order to reduce the number of elements and hence the computation time. The final number of elements was approximately 180.000 .
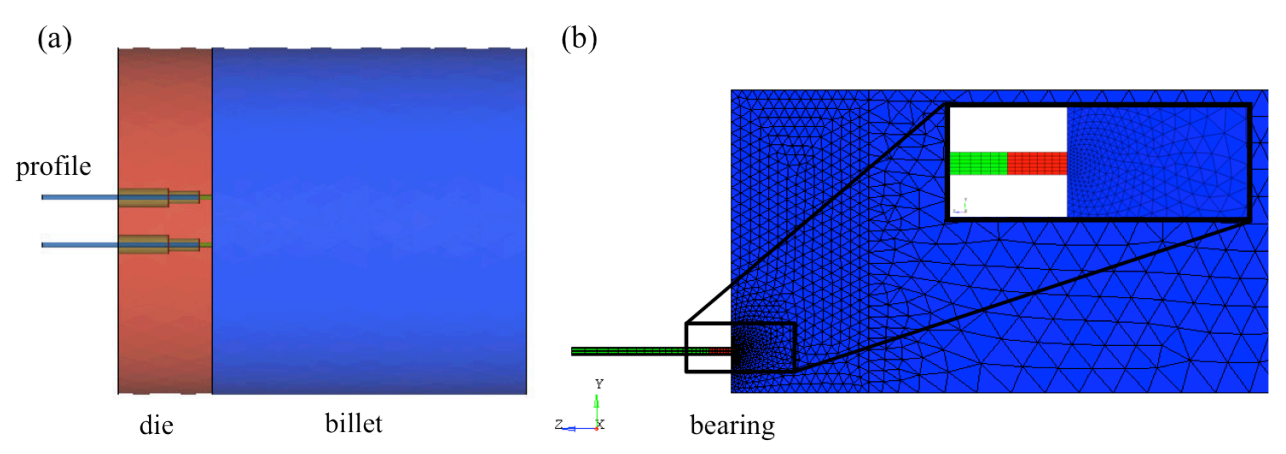

FIGURE 2. Illustration of the extrusion model, (a) 3D-model, (b) mesh of the workpiece in 2D

The thermal visco-plastic behaviour of the magnesium alloys is described by the constitutive relationship using the hyperbolic sine form $[5,6]$ :

$$
\dot{\varepsilon} \exp \left(\frac{Q}{R T}\right)=Z=A(\sinh \alpha \sigma)^{n},
$$

where $Z$ is the temperature compensated strain rate, $A$ and $n$ material constants, $\alpha$ the stress multiplier, $\dot{\varepsilon}$ the strain rate, $Q$ the apparent activation energy, $R$ the universal gas constant and $T$ the absolute temperature. The material parameters over a wide range of temperatures and strain rates were determined using hot compression tests and were already published in a previous work [6]. The process and material parameters are summarized in TABLE 1.

While a heat transfer coefficient of $5000 \mathrm{Wm}^{-2} \mathrm{~K}^{-1}$ was used at the tool-workpiece interface the heat transfer at the profile-air interface was idealized using convection with a value of $20 \mathrm{Wm}^{-2} \mathrm{~K}^{-1}$. The verification of presimulation tests with experimental trials (see in [3]) showed, that the visco-plastic friction model is most suitable to simulate the profile-bearing interface. Here, the visco-plastic friction model corresponds to the Coulomb friction model, where the friction shear stress $\tau=f\left(p, \sigma_{f}, \mu\right)$ is a function of the pressure and the flow stress. The factor $\mathrm{m}$ was set to 0,3 in order to scale the required shear stress. In contrast, the contact zone between container and billet is idealized by non-friction and adhesion respectively. 
TABLE (1). Process and material parameters

\begin{tabular}{lcc}
\hline Parameters & Magnesium alloy AZ31 & Magnesium alloy ME21 \\
\hline Billet diameter & $110 \mathrm{~mm}$ & 110 \\
Double die orifice & $(2) \times 80 \mathrm{~mm} \times 1.5 \mathrm{~mm}$ & $(2) \times 80 \mathrm{~mm} \times 1.5 \mathrm{~mm}$ \\
Extrusion ratio & $39: 1$ & $39: 1$ \\
Ram speed & $1.5 \mathrm{mms}^{-1}$ & $1.5 \mathrm{mms}^{-1}$ \\
Extrusion temperature & $300{ }^{\circ} \mathrm{C}$ & $300{ }^{\circ} \mathrm{C}$ \\
& & \\
Density & $1.77 \mathrm{kgm}^{-3}$ & $1.77 \mathrm{kgm}^{-3}$ \\
Thermal expansion & $0.0000279 \mathrm{~K}^{-1}$ & $0.0000279 \mathrm{~K}^{-1}$ \\
Poison ratio & 0.35 & 0.35 \\
Heat conductivity & $76.9 \mathrm{Wm}^{-1} \mathrm{~K}^{-1}$ & $76.9 \mathrm{Wm}^{-1} \mathrm{~K}^{-1}$ \\
Specific heat & $980 \mathrm{Jkg}^{-1} \mathrm{~K}^{-1}$ & $980 \mathrm{Jkg}^{-1} \mathrm{~K}^{-1}$ \\
& & \\
Young modulus & $45 \mathrm{GPa}^{-1}$ & $45 \mathrm{GPa}^{-1}$ \\
Stress multiplier $\alpha$ & $0.010 \mathrm{MPa}^{-1}$ & $0.023 \mathrm{MPa}^{-1}$ \\
Activation energy Q & $108000 \mathrm{Jmol}^{-1}$ & $182000 \mathrm{Jmol}^{-1}$ \\
Constant $n$ & 6.3 & 4.7 \\
Constant A & $4.9 \mathrm{E}+08$ & $3.5 \mathrm{E}+11$ \\
\hline
\end{tabular}

\section{RESULTS AND DISCUSSION}

An experimental study revealed that the sheet extrusion of the rare earth magnesium alloy ME21 produces pronounced buckling, waving as well as variations in profile geometry compared to the extrusion of the commercial magnesium alloy AZ31. Especially the sheet extrusion using low billet temperatures leads to distinctive buckling directly after passing the die orifice (see in [7]).

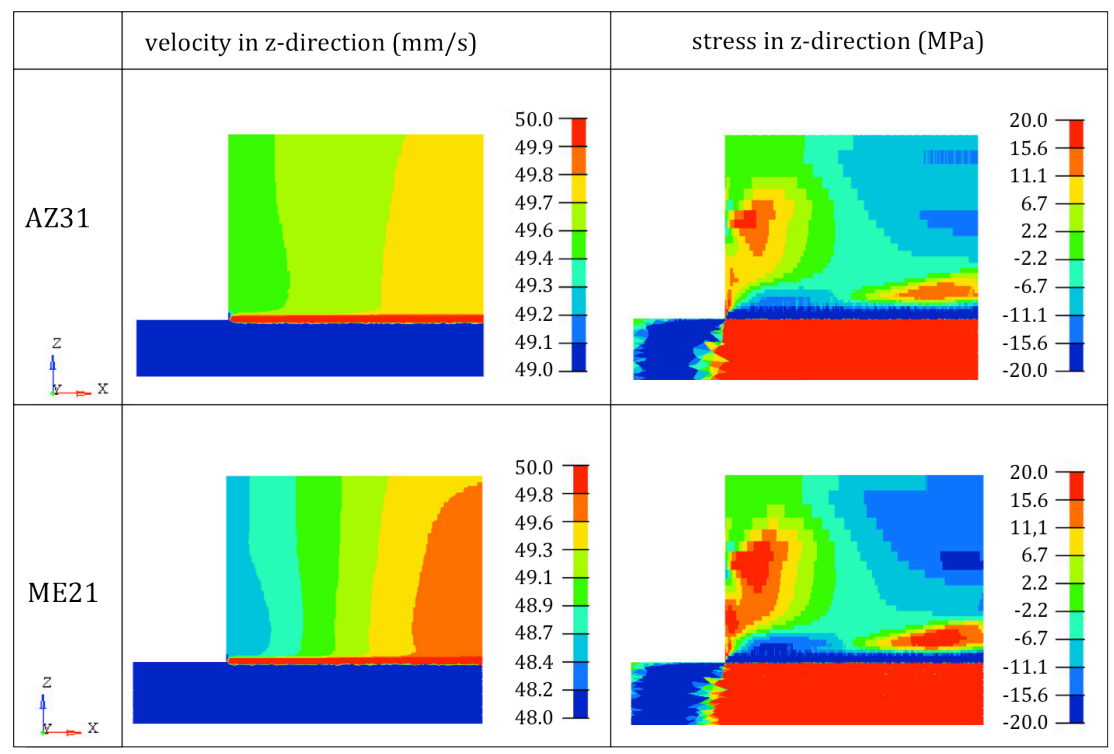

FIGURE 3. Velocity and stress distribution along the extrusion direction (z-direction) of the magnesium alloys AZ31 and ME21 at an initial temperature of $300{ }^{\circ} \mathrm{C}$ for the steady state extrusion (time: $80 \mathrm{~s}$ )

In order to identify the origin of the mentioned phenomena the sheet extrusion of both magnesium alloys AZ31 and ME21 was simulated at an initial temperature of $300^{\circ} \mathrm{C}$. FIGURE 3 exhibits the velocity distribution as well as the stress distribution in the exit profile along the extrusion direction (z-direction) for the steady state extrusion (time: $80 \mathrm{~s}$ ) for both magnesium alloys. Both alloys show the same tendency in the velocity and stress distribution. in the centre of the extruded sheet a higher velocity than at the edge of the profile occurs resulting in a material flow 
gradient, which is stronger developed for the ME21 sheet extrusion. The inhomogeneous material flow causes a stress gradient along the profile widths, which is characterized by a compression stress zone in the centre as well as a tension stress zone at the edge of the sheets. The compression stress zone results from the flow imbalance, because the slower flowing material at the edge restricts the material in the centre featuring a high flow velocity. Consequently the near edge material zone exhibits tension stresses. The stronger pronounced flow imbalance in case of ME21 extrusion results in larger stresses. After passing the die orifice buckling of the sheet appears if the compression stresses exceed a critical value [4]. This critical value depends on the spatial velocity and stress distribution. If the compression stress zone is extended larger than the tensile stress zone buckling of the sheet is most probable [4]. Due to the development of more extended compression stresses in the ME21 profile a stronger waving as well as a more pronounced buckling appears.

The flow imbalance does not only influence the formation of waving and buckling during the extrusion of the magnesium sheets, but also affects the profile geometry. FIGURE 4 presents the velocity, equivalent strain and temperature distributions of the sheet cross-section in the bearing length of the die for both magnesium alloys. The friction between the profile and the bearing causes decreasing flow velocities accompanied by increasing equivalent strains along the cross section, which is most pronounced near the contact zone. Here, in the centre region of the profile the gradient of the equivalent strain is developed over a larger region than at the edge of the profile. This finding is related to both the flow imbalance in the extrusion direction and the flow imbalance along the cross section due to the friction leading to underfilling of the die bearing. This results in a variation of the exit profile geometry, where in the centre a more distinctive variance in the profile shape is found than at the edge [7]. The development of a temperature gradient along the cross section additionally enhances the imbalance in material flow and the gradient of the equivalent. In agreement with the simulation results the ME21-sheets deliver a quantitatively larger variance in profile accuracy than the AZ31-sheets [7].

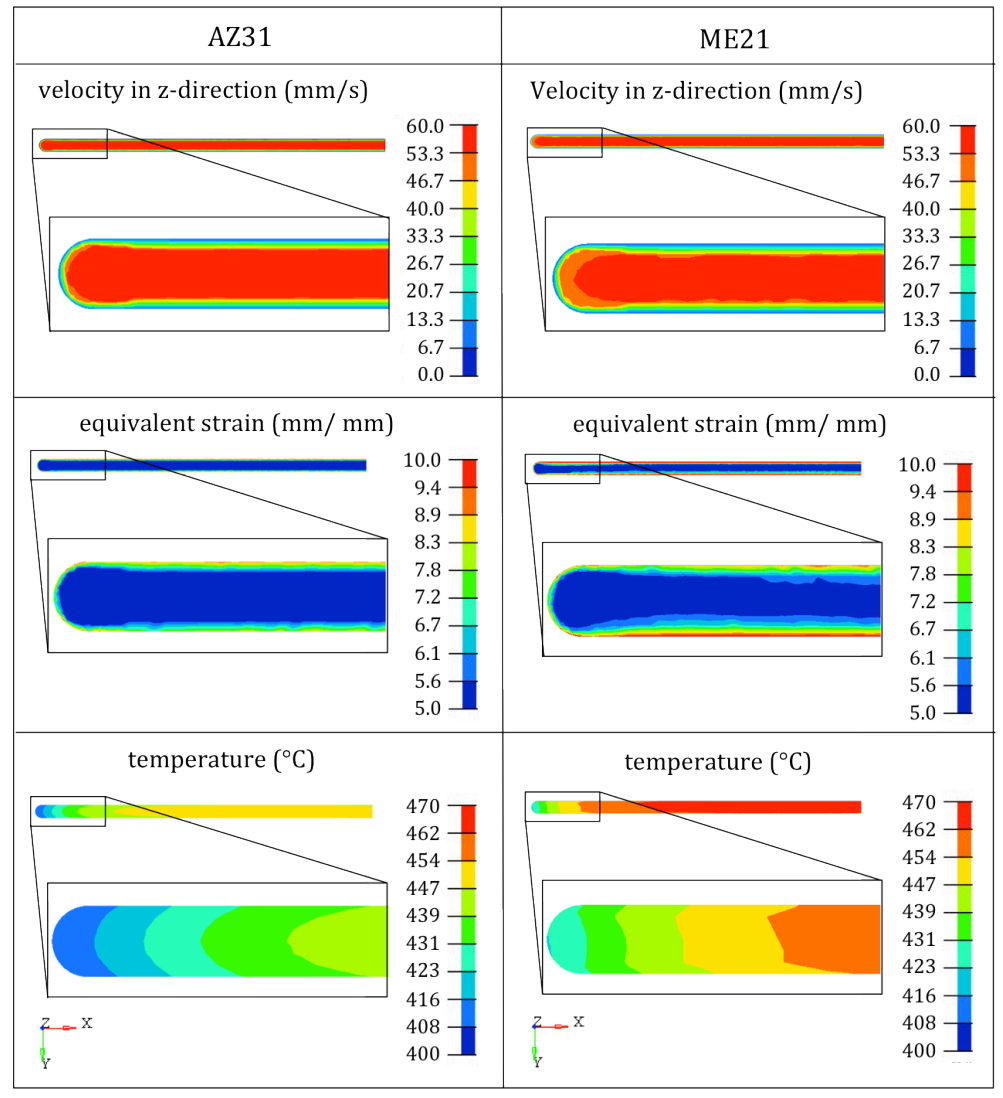

FIGURE 4. Velocity, strain and temperature distribution along the sheet cross section in the die bearing of the magnesium alloys AZ31 and ME21 at an initial temperature of $300{ }^{\circ} \mathrm{C}$ for the steady state extrusion (time: $80 \mathrm{~s}$ ) 


\section{CONCLUSIONS}

The simulation of magnesium sheet extrusion trials show that due to the material flow imbalance a large compression stress zone is formed causing buckling and waving of the thin sheets. Furthermore, the simulations

reveal that the interaction of the material flow gradients along the width and along the thickness direction near the die orifice lead to inaccuracies of the profile shape.

\section{ACKNOWLEDGEMENT}

The authors are grateful for the financial support from the Deutsche Forschungsgemeinschaft (DFG) under the contract number MU 2963/6-1.

\section{REFERENCES}

1. E. Aghion and B. Bronfin, Mater. Sci. Forum 350-351, 19-28 (2005).

2. M. C. Kulekci, J. Adv. Manuf. Technol. 39, 851-865 (2008).

3. S. Gall, S. Müller, W. Reimers, Int. J. Mater. Form. 6, 187-197 (2013).

4. F. Halvorsen and T. Aukrust, Int. J. Plasticity 22, 158-173 (2006).

5. H. J. McQueen and N. D. Ryan, Mater. Sci. Eng. A 322, 43-63 (2002).

6. S. Gall, M. Huppmann, H. M. Mayer, S. Müller, W. Reimers, J. Mater. Sci. 48, 473-480 (2013).

7. S. Gall, "Grundlegende Untersuchungen zum Strangpressen von Magnesiumblechen und deren Weiterverarbeitung: Mikrostruktur und mechanische Eigenschaften”, Ph.D. Thesis, Technische Universität Berlin, 2013. 